\title{
Blunt cerebrovascular injury in the geriatric population
}

\author{
Diana T. Le, MS, ${ }^{1}$ Kinsey A. Barhorst, BS, ${ }^{1}$ James Castiglione, BS, ${ }^{1}$ George L. Yang, MD, ${ }^{2}$ \\ Sanjit J. Shah, MD, ${ }^{2}$ Sarah S. Harlan, PharmD, ${ }^{3}$ Shaun P. Keegan, PharmD, BCPS, ${ }^{3}$ \\ Roman A. Jandarov, PhD, ${ }^{4}$ Laura B. Ngwenya, MD, PhD, ${ }^{2}$ and Charles J. Prestigiacomo, MD ${ }^{2}$ \\ ${ }^{1}$ University of Cincinnati College of Medicine; ${ }^{2}$ Department of Neurological Surgery, University of Cincinnati College of Medicine; \\ ${ }^{3}$ Division of Pharmaceutical Sciences, James L. Winkle College of Pharmacy, University of Cincinnati; and ${ }^{4}$ Division of Biostatistics \\ and Bioinformatics, Department of Environmental Health, University of Cincinnati College of Medicine, Cincinnati, Ohio
}

\begin{abstract}
OBJECTIVE Blunt cerebrovascular injury (BCVI) is associated with high rates of neurological morbidity and mortality. The detection and management of BCVI has improved with advances in imaging and sensitive screening protocols. Few studies have explored how these injuries specifically affect the geriatric population. The purpose of this retrospective analysis was to investigate the presentation and prognosis of BCVI in the elderly population and to assess its clinical implications in the management of these patients.
\end{abstract}

METHODS All patients presenting to the University of Cincinnati (UC) level I trauma center between February 2017 and December 2019 were screened for BCVI and entered into the prospectively maintained UC Neurotrauma Registry. Patients with BCVI confirmed by CT angiography underwent retrospective chart reviews to collect information regarding demographics, positive screening criteria, cause of injury, antithrombotic agent, injury location, Denver Grading Scale, hospital and ICU length of stay, and discharge disposition. Patients were divided into geriatric (age $\geq 65$ years) and adult (age < 65 years) subgroups. Continuous variables were analyzed using the Student t-test and categorical variables with the Pearson chi-square test.

RESULTS Of 124 patients with BCVI, stratification by age yielded 23 geriatric and 101 adult patients. Injury in the geriatric group was associated with significantly higher mortality $(p=0.0194)$. The most common cause of injury in the elderly was falls $(74 \%, 17 / 23 ; p<0.0001)$, whereas motor vehicle accidents were most common in the adult group $(38 \%, 38 / 100$; $p=0.0642)$. With respect to the location of injury, carotid $(p=0.1171)$ and vertebral artery $(p=0.6981)$ injuries did not differ significantly for the geriatric group. The adult population presented more often with Denver grade I injuries $(p<$ $0.0001)$, whereas the geriatric population presented with grade IV injuries $(p=0.0247)$. Elderly patients were more likely to be discharged to skilled nursing facilities $(p=0.0403)$ and adults to home or self-care $(p=0.0148)$.

CONCLUSIONS This study is the first to characterize BCVI to all cervical and intracranial vessels in the geriatric population. Older age at presentation is significantly associated with greater severity, morbidity, and mortality from injury, with no preference for the particular artery injured. These findings carry important clinical implications for adapting practice in an aging population.

https://thejns.org/doi/abs/10.3171/2020.7.FOCUS20499

KEYWORDS BCVI; blunt cerebrovascular injury; geriatrics

$\mathrm{B}$ LUNT cerebrovascular injury (BCVI) is nonpenetrating injury to the vertebral or carotid arteries due to major or minor trauma. Although early reports suggest only $0.1 \%$ of the trauma population suffer BCVI, improvements in vascular imaging have increased detection and incidence rates to $2 \%-3 \%,{ }^{1,2}$ Early diagnosis of and intervention for BCVI is imperative because $10 \%-$ $20 \%$ of lesions $^{3}$ can result in ischemia and infarction, with subsequent neurological morbidity and mortality rates of
$80 \%$ and $40 \%$, respectively. ${ }^{2}$ Recent studies have demonstrated dramatic decreases in the rates of ischemic stroke as a result of improved imaging, screening protocols, and interventions-from $64 \%$ to as low as $0.3 \%-6.8 \% .4,5$

Given the increasing prevalence of BCVI in the trauma population, attention has shifted to this subject over the past 2 decades. Biffl et al. first described several criteria to screen patients for BCVI based on mechanism of injury $^{6}$ (Appendix A). After subsequent revisions, they are 
now known as the expanded Denver criteria and have been widely accepted by the trauma community. Of these injury mechanisms, the most common causes of BCVIs in adults were motor vehicle accidents, whereas geriatric patients more commonly presented due to falls from standing height. ${ }^{7,8}$ With the population of both the United States and the international community aging rapidly, and 1 in every 5 people expected to be older than 65 years by $2030,{ }^{9}$ special attention should be given to the geriatric population.

Limited information exists to describe the effects of BCVI on the population of adults older than 65 years of age. Page and Josiah demonstrated that mortality specifically following vertebral artery injuries was significantly higher in the geriatric age group than in the general population. ${ }^{8}$ Additional studies have shown that institutions often miss BCVI in the geriatric population, but even when screened appropriately, detection of the injury does change management of the patient's injuries. ${ }^{7}$ Still, a more thorough evaluation of the geriatric population is needed. Our goal was to characterize the prevalence, mechanisms, etiology, and outcomes of all forms of BCVI in the geriatric population.

\section{Methods}

All patients presenting to the University of Cincinnati (UC) level I trauma center between February 2017 and December 2019 were screened for BCVI and entered into the prospectively maintained UC Neurotrauma Registry. Screening criteria for BCVI included a set of evidencebased signs, symptoms, and mechanisms of injury predetermined by our institution ${ }^{10}$ and based on BCVI Practice Guidelines established by the Eastern Association for the Surgery of Trauma (EAST) (Appendix B). ${ }^{2}$ Injuries were confirmed and characterized by a trained neuroradiologist with CT angiography. Patients meeting the criteria for BCVI were entered into the database. Data were collected and managed using REDCap electronic data capture tools hosted at the UC. REDCap is a secure, web-based software platform designed to support data capture for research studies. ${ }^{11,12}$

The UC Neurotrauma Registry was queried on March 24,2020 , to obtain all patients with BCVI. Exclusion criteria were nontraumatic or spontaneous injury, penetrating injuries, and age younger than 15 years. Complete retrospective chart reviews were performed to collect demographic information, positive screening criteria, etiology, injury location (Fig. 1), Denver Grading Scale (Appendix A), hospital and ICU length of stay, anticoagulant or antiplatelet therapy, stroke documented on imaging, and discharge disposition. Mortality was defined as in-hospital death. Patients were divided into geriatric (age $\geq 65$ years) and adult (age $<65$ years) subgroups.

\section{Statistical Analysis}

All values are reported as the mean $\pm \mathrm{SD}$ for continuous variables. Data within the paper are calculated using the total number of patients in each age group in the denominator, unless otherwise stated. Tables illustrate percentages and significance based on a denominator of the characteristic being assessed, depicted by rows. Simple univariate statistical analysis was performed using Microsoft Excel, whereas multivariate analysis was conducted by the department's statistician using R (https://www.r-project.org/). Continuous variables were analyzed with the Student t-test and categorical variables with Pearson's chi-square test. A $\mathrm{p}$ value $<0.05$ was defined as significant. Institutional review board approval for the study was sought and granted.

\section{Results}

Of 1345 patients in the registry, 124 met the criteria for BCVI in the defined time period. The results are shown in Table 1. The incidence of BCVI in our neurotrauma population was $9.2 \%$. Stratification by age yielded 23 patients in the geriatric group (mean age $75.30 \pm 7.12$ years, median age 75 years) and 101 patients in the adult group (mean age $38.16 \pm 12.89$ years, median age 37 years).

Age as a continuous variable was significantly associated with higher mortality $(p=0.0194)$, but significance was not preserved with analysis as a categorical variable $(\mathrm{p}=$ 0.1921). For all patients, carotid injuries were associated with greater mortality than vertebral artery injuries $(\mathrm{p}=$ 0.0290 ), although there was no difference between the two vessels in stroke documented on imaging ( $p=0.7983)$. Of every combination of Denver Grading Scale and arterial region (Fig. 1), a grade IV injury to the left internal carotid artery in the upper cervical region was the only one that was significantly associated with mortality $(p=0.0395)$. Importantly, no significant associations between antiplatelet therapy and mortality $(\mathrm{p}=0.3836)$ nor between anticoagulation therapy and mortality $(\mathrm{p}=0.6667)$ were found.

\section{Subgroup Analysis}

\section{Demographics}

The geriatric and adult BCVI groups demonstrated incidence rates of $4.7 \%$ and $11.8 \%$, respectively. Adult patients were primarily males $(75 \%)$, whereas there was near 1:1 gender distribution in the geriatric group at 52.2\% male and $47.8 \%$ female (12/23 and $11 / 23$, respectively). Although the overall number of males exceeded that of females (87 and 36, respectively) (Table 1), there was no statistically significant difference in gender in the adult group $(p=0.3482)$ or in the geriatric group $(p=0.0504)$.

\section{Etiology and Screening}

Despite the greater prevalence of falls in the adult population $(58.5 \%$ ) (Table 2 ), $74 \%$ of the older population presented status post-fall $(17 / 23, \mathrm{p}<0.0001)$. The vast majority $(90.5 \%)$ of motor vehicle accidents occurred in the younger age group (Table 2), and this was likewise the most common cause of injury in that group (38/100,38\%; $\mathrm{p}=0.0642$ ). In the geriatric group, falls from stairs or standing height accounted for $41.2 \%$ and $35.3 \%$ of fall injuries, respectively. Polytrauma accounted for $87.5 \%$ of injuries in both groups. There was no significant difference in presenting signs, symptoms, or mechanism of injury as assessed by our institution's screening criteria (Table 3).

\section{Location and Severity}

A preference for injuries occurring to the carotid or vertebral artery was not found for the geriatric group $(\mathrm{p}=$ $0.3759)$. In total, there were 11 carotid and 14 vertebral ar- 


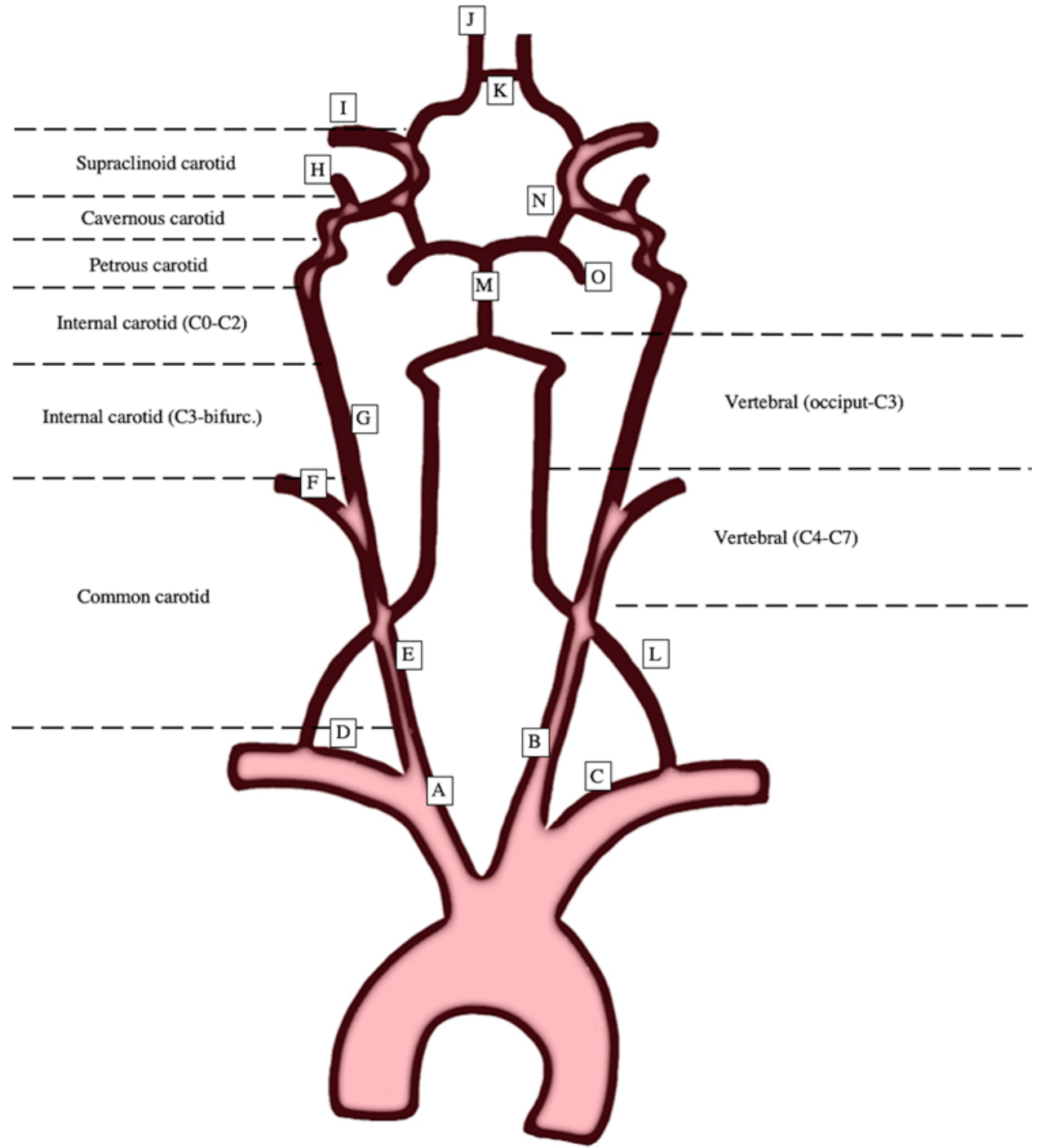

FIG. 1. Subcategorizations of arterial sites of BCVI. $A=$ brachiocephalic artery; $B=$ left common carotid artery; bifurc. = bifurcation; $C=$ left subclavian artery; $D=$ right subclavian artery; $E=$ right common carotid artery; $F=$ right external carotid artery; $G=$ right internal carotid artery; $\mathrm{H}=$ right ophthalmic artery; I = right middle cerebral artery; $\mathrm{J}=$ right anterior cerebral artery; $\mathrm{K}=$ anterior communicating artery; $\mathrm{L}=$ left vertebral artery; $\mathrm{M}=$ basilar artery; $\mathrm{N}=$ left posterior communicating artery; $\mathrm{O}=$ left posterior cerebral artery. Copyright Diana T. Le. Published with permission.

tery injuries in the geriatric group. Similarly, there was no preference for carotid or vertebral artery in the adult group $(\mathrm{p}=0.3298)$. The adult group had 65 carotid and 56 total vertebral artery injuries. With regard to severity of injury, adults were significantly more likely to present with Denver grade I injuries $(\mathrm{p}<0.0001)$ and elderly patients with grade IV injuries ( $p=0.0247)$ (Figs. 2 and 3 ).

\section{Outcomes}

The most common outcomes overall were death, home or self-care, long-term care, and rehabilitation facility (Table 4). Dispositions for geriatric patients were most frequently death $(7 / 23,30.4 \%)$ or discharge to skilled nursing facilities $(7 / 23,30.4 \% ; \mathrm{p}=0.0280)$; adult patients were likely to be discharged to home or self-care $(27 / 100,27 \%$; $\mathrm{p}=0.0127)$. No significant difference in the hospital ( $\mathrm{p}$
$=0.1808)$ or ICU length of stay $(p=0.3388)$ was found between groups. The presence and absence of stroke on imaging tended to be more common in the adult group by numbers alone $(85.7 \%$ and $81.6 \%$, respectively) (Table 4 ), but there was no significant difference in documented stroke on imaging for the two age groups $(p=0.7646)$.

\section{Discussion}

This study is the first to characterize the entire scope of BCVI in the geriatric population, analyzing both carotid and vertebral artery injuries. Older age at presentation is associated with greater severity, morbidity, and mortality from injury, with no preference for the particular artery injured. Prior work by Page and Josiah describes traumatic vertebral artery injuries in the geriatric population. ${ }^{8}$ The 
TABLE 1. Demographics of the BCVI population by adult and geriatric groups

\begin{tabular}{|c|c|c|c|c|}
\hline Category & No. & Adult* & Geriatric $†$ & $p$ Value \\
\hline \multicolumn{5}{|l|}{ Sex } \\
\hline Male & 87 & $75(86.2 \%)$ & $12(13.8 \%)$ & 0.2405 \\
\hline Female & 36 & $25(69.4 \%)$ & $11(30.6 \%)$ & 0.0681 \\
\hline \multicolumn{5}{|l|}{ Race } \\
\hline Asian & 0 & 0 & 0 & NA \\
\hline $\begin{array}{c}\text { Black or African } \\
\text { American }\end{array}$ & 13 & $11(84.6 \%)$ & $2(15.4 \%)$ & 0.7592 \\
\hline Hispanic & 5 & $5(100 \%)$ & 0 & 0.2835 \\
\hline White or Caucasian & 103 & $83(80.6 \%)$ & $20(19.4 \%)$ & 0.8517 \\
\hline Other & 2 & $1(50 \%)$ & $1(50 \%)$ & 0.2562 \\
\hline \multicolumn{5}{|l|}{ Marital status } \\
\hline Married & 39 & $27(69.2 \%)$ & $12(30.8 \%)$ & 0.0532 \\
\hline Single & 52 & $48(92.3 \%)$ & $4(7.7 \%)$ & 0.0418 \\
\hline Legally separated & 2 & $2(100 \%)$ & 0 & 0.4976 \\
\hline Divorced & 12 & $9(75 \%)$ & $3(25 \%)$ & 0.5756 \\
\hline Widowed & 3 & 0 & $3(100 \%)$ & 0.0003 \\
\hline Unspecified & 6 & $6(100 \%)$ & 0 & 0.2401 \\
\hline Unknown & 6 & $5(83.3 \%)$ & $1(16.7 \%)$ & 0.8984 \\
\hline Other & 0 & 0 & 0 & NA \\
\hline Significant other & 3 & $3(100 \%)$ & 0 & 0.4062 \\
\hline \multicolumn{5}{|l|}{ Financial class $\ddagger$} \\
\hline Commercial & 12 & $12(100 \%)$ & 0 & 0.1037 \\
\hline Managed Medicare & 4 & 0 & $4(100 \%)$ & $<0.0001$ \\
\hline Managed Medicaid & 17 & $17(100 \%)$ & 0 & 0.0528 \\
\hline Medicaid & 3 & $3(100 \%)$ & 0 & 0.4159 \\
\hline Medicare & 11 & $4(36.4 \%)$ & $7(63.6 \%)$ & $<0.0001$ \\
\hline Pending Medicaid & 8 & $8(100 \%)$ & 0 & 0.1840 \\
\hline Other government & 3 & $3(100 \%)$ & 0 & 0.4159 \\
\hline Other nongovernment & 0 & 0 & 0 & NA \\
\hline Self-pay & 25 & $21(84 \%)$ & $4(16 \%)$ & 0.7877 \\
\hline \multicolumn{5}{|l|}{ Employment status } \\
\hline Disabled & 6 & $6(100 \%)$ & 0 & 0.2401 \\
\hline Full time & 30 & $28(93.3 \%)$ & $2(6.7 \%)$ & 0.0910 \\
\hline Non-employed & 51 & $49(96.1 \%)$ & $2(3.9 \%)$ & 0.0068 \\
\hline Part time & 7 & $7(100 \%)$ & 0 & 0.2045 \\
\hline Retired & 17 & 0 & $17(100 \%)$ & $<0.0001$ \\
\hline Self-employed & 4 & $3(75 \%)$ & $1(25 \%)$ & 0.7465 \\
\hline Student, full time & 4 & $4(100 \%)$ & 0 & 0.3375 \\
\hline Student, part time & 0 & 0 & 0 & NA \\
\hline Active military & 0 & 0 & 0 & NA \\
\hline Unknown & 4 & $3(75 \%)$ & $1(25 \%)$ & 0.7465 \\
\hline Unspecified & 0 & 0 & 0 & NA \\
\hline
\end{tabular}

NA = not applicable.

Percentages are reported with a denominator of the total number of patients in each variable (represented in rows). Boldface type indicates statistical significance.

* Adult group included 101 patients < 65 years old (mean age $38.16 \pm 12.89$ years); age was statistically significant, at $p=0.0194$ (continuous variable)

Demographic data were unavailable for 1 patient in this group.

† Geriatric group included 23 patients $\geq 65$ years old (mean age $75.30 \pm 7.12$ years); age was not statistically significant, at $p=0.1921$ (categorical variable). $\ddagger$ Financial data were available for 83 patients.
TABLE 2. Etiology of BCVI by age group

\begin{tabular}{lrclc}
\hline \multicolumn{1}{c}{ Variable } & Overall & Adult & Geriatric & p Value \\
\hline Cause of injury, no. & & & & \\
\hline All-terrain vehicle & 2 & $1(50 \%)$ & $1(50 \%)$ & 0.2562 \\
\hline Assault & 3 & $2(66.7 \%)$ & $1(33.3 \%)$ & 0.5156 \\
\hline Fall & 41 & $24(58.5 \%)$ & $17(41.5 \%)$ & $\mathbf{0 . 0 0 0 2}$ \\
\hline Fall from moving object & 0 & 0 & 0 & $\mathrm{NA}$ \\
\hline Gunshot wound & 0 & 0 & 0 & $\mathrm{NA}$ \\
\hline Motor vehicle accident & 42 & $38(90.5 \%)$ & $4(9.5 \%)$ & 0.1272 \\
\hline Motorcycle accident & 11 & $11(100 \%)$ & 0 & 0.1117 \\
\hline Other & 5 & $5(100 \%)$ & 0 & 0.2835 \\
\hline Pedestrian struck & 16 & $16(100 \%)$ & 0 & 0.0551 \\
\hline Struck by object & 3 & $3(100 \%)$ & 0 & 0.4062 \\
\hline Unknown & 1 & $1(100 \%)$ & 0 & $\mathrm{NA}$ \\
\hline
\end{tabular}

Percentages are reported with a denominator of the total number of patients in each variable (represented in rows). Boldface type indicates statistical significance.

differences in patient selection and arterial inclusion between their study and ours expand the understanding of geriatric BCVI. To identify patients, the authors of the previous study cross-referenced all traumatic cervical spine injuries with patients found to have a vertebral artery injury. Our study, however, screened all trauma patients presenting to our institution by using predefined UC Neurotrauma BCVI screening criteria. ${ }^{2,10}$

The overall incidence of BCVI in our population was 9.2\%. A 7-year prospective study performed at the SainteAnne Military Hospital of Toulon, France, similarly reported a $9.2 \%$ incidence rate of BCVI, although there are differences in methodology between studies. Their study included only patients admitted with severe traumatic brain injury, ${ }^{13}$ whereas our database includes all patients with any neurotrauma. The similar incidence rates could be accounted for by sensitive screening criteria that might have facilitated detection of BCVI in milder traumatic brain injury. These criteria were created and jointly implemented by the trauma and neurosurgery departments at our institution in February 2018 (Appendix B). The methods used to identify patients in the current study might be more representative of clinical scenarios in which all patients are screened using a multidisciplinary, evidencebased set of signs and symptoms. We are confident that the patients included in this study capture the true population of BCVI in the Greater Cincinnati/Northern Kentucky area because this institution represents the only level I trauma center in the region.

This study expands on previous work by including carotid as well as vertebral artery injuries. When both age groups were combined, carotid artery injuries were associated with a significantly higher risk of mortality, although with no difference in documented stroke. Ho et al. similarly found no significant association in stroke between the anterior and posterior circulation following BCVI. ${ }^{14}$ Furthermore, in our study the particular combination of a grade IV injury to the left internal carotid artery in the upper cervical region $(\mathrm{C} 0-2)$ was found to 
TABLE 3. University of Cincinnati BCVI screening criteria by age group

\begin{tabular}{lrrrr}
\hline \multicolumn{1}{c}{ Variable } & Overall & Adult & Geriatric & p Value \\
\hline Mechanism, no. & & & & \\
\hline High-energy impact & 49 & 42 & 7 & 0.6010 \\
\hline Le Fort Il or III fracture & 4 & 4 & 0 & 0.3637 \\
\hline Skull base fracture & 45 & 35 & 10 & 0.3613 \\
\hline Diffuse axonal injury & 3 & 3 & 0 & 0.4315 \\
\hline High C-spine injury (occipital condyle, C1-3) & 33 & 26 & 7 & 0.5302 \\
\hline Cervical vertebral body fracture or fracture through foramen & 32 & 26 & 6 & 0.8040 \\
$\quad$ transversarium & & & & \\
\hline Subluxation or ligamentous injury at any level & 11 & 9 & 2 & 0.9240 \\
\hline Significant thoracic/cardiac blunt-force trauma & 16 & 15 & 1 & 0.2491 \\
\hline Signs \& symptoms, no. & & & & \\
\hline Arterial bleeding & 9 & 6 & 3 & 0.3351 \\
\hline Audible neck bruit & 1 & 1 & 0 & 0.6128 \\
\hline Skull base fracture & 49 & 42 & 7 & 0.2890 \\
\hline High C-spine injury & 36 & 27 & 9 & 0.4922 \\
\hline Expanding neck hematoma & 4 & 3 & 1 & 0.8189 \\
\hline Focal neurological signs & 4 & 3 & 1 & 0.8189 \\
\hline
\end{tabular}

be significantly correlated with mortality. The cervical internal carotid artery has been described to be especially vulnerable in the region between the petrous canal and soft tissue of the neck. ${ }^{3}$ The liability of this region to blunt injury might be explained by the transitional anatomy. The petrous portion of the carotid artery is bound proximally by a fibrous ring ${ }^{15}$ that, with an external force, can act as an anchor superiorly while more inferior aspects move freely. Likewise, the distal segment of the vertebral artery represents another transition point. The portion entering the dura mater $\left(\mathrm{V}_{3}\right.$ segment $\left.^{16}\right)$ subsequently adheres to a fibrous ring, and proximal segments travel a tortuous vertical and horizontal course with exposures between transverse foramina and the foramen magnum. Blunt-force injury and subsequent rotation, flexion, and hyperextension of the neck in these more mobile areas with 1-sided anatomical tethers can result in stretch injuries and vessel stenosis. Coincidingly, grade I injuries were found to be most common in the carotid upper cervical region and vertebral C3-occiput region.

The association of injury severity with the left carotid artery, in particular, may be explained by anatomical asymmetry. In general, the right carotid artery branches from the innominate or brachiocephalic artery, which emerges from the right subclavian artery, whereas the left carotid artery branches directly from the left subclavian artery. Although anatomical variation is to be expected, an asymmetrical course can understandably give rise to laterality in susceptibility to injury. The more inferior branch point of the left side is analogous to a lower "tethering" point; the longer course permits hypermobility of the left side during blunt trauma, resulting in greater injury and subsequent endothelial reactions and stenosis. A study by Golemati et al. ${ }^{17}$ confirmed significant asymmetry in the biomechanical and textural features between the left and right carotid arteries by using ultrasound. The differences

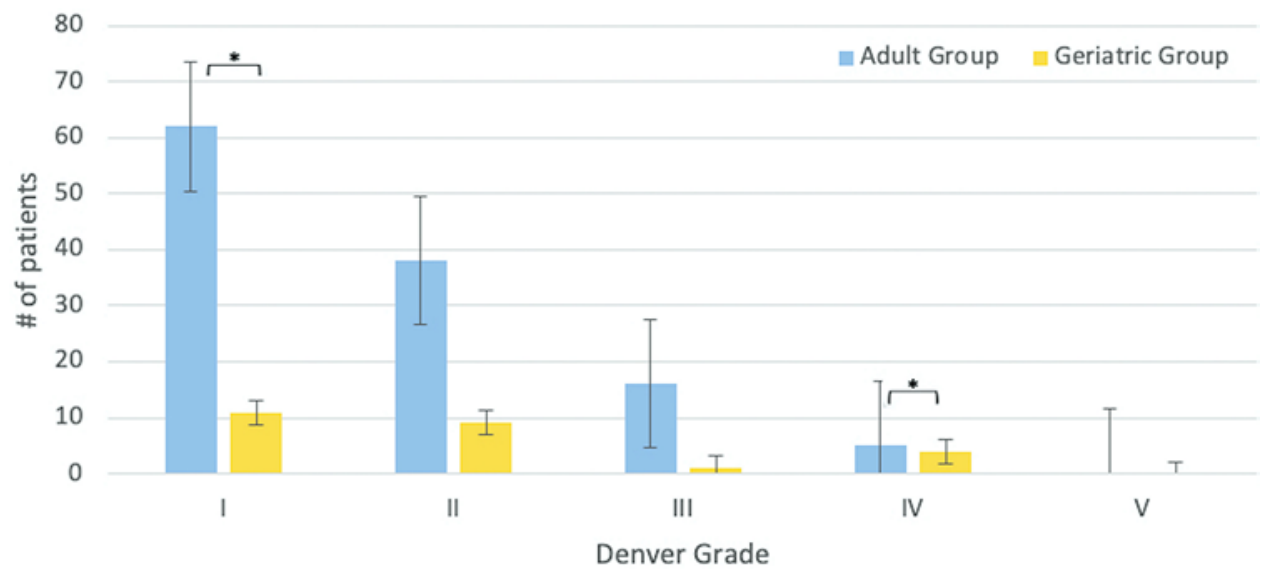

FIG. 2. Severity of BCVI by age group. *Significant at $p<0.05$. 


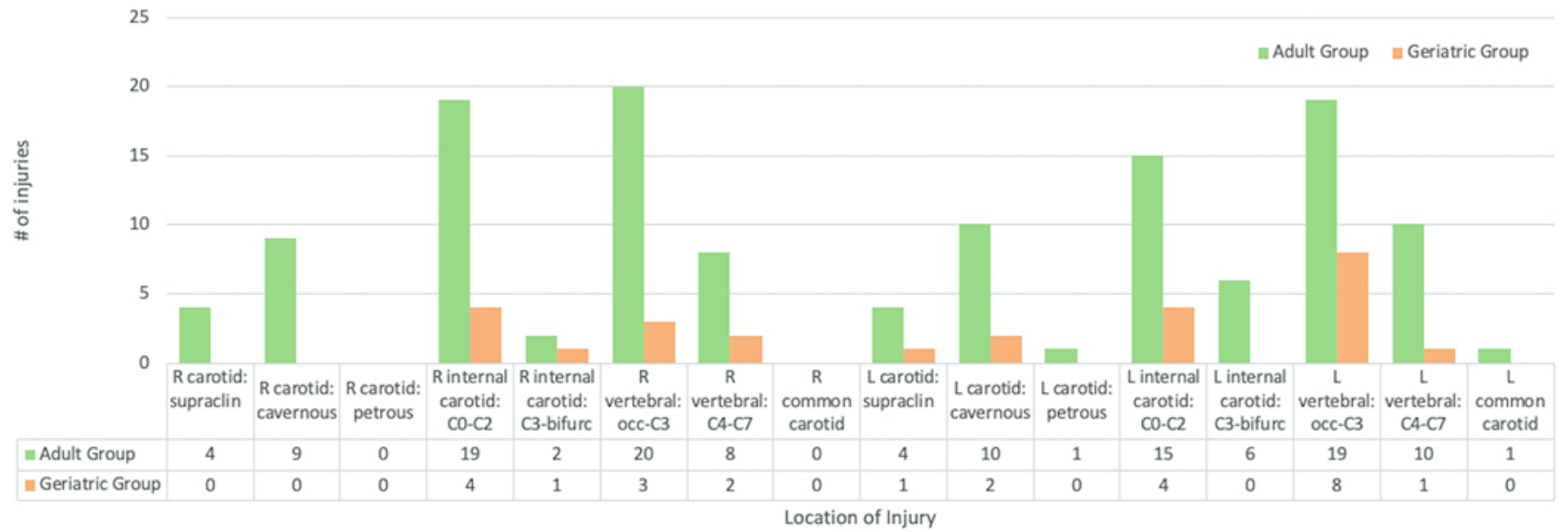

FIG. 3. Distribution of BCVI by age group and location. Occ = occiput; supraclin = supraclinoid.

were found to be primarily mechanical in nature, most notably in entropy of radial velocity, longitudinal strain index, and total diastole-to-systole displacement. ${ }^{17}$ Given the asymmetrical course of the carotid artery and significant mortality associated with left-sided grade IV upper cervical injuries, the inclusion of the carotid artery is essential for thorough study of BCVI.

\section{Geriatric Analysis}

Age was significantly associated with increased mortality due to BCVI when analyzed as a continuous variable; interestingly, categorical analysis with a threshold at age 65 years did not preserve the significance. The traditional definition of old age as beginning in the 6th or 7th decade of life may be outdated. A decline in trauma outcomes has been documented as early as the age of 45 years..$^{18}$ Additionally, the odds of dying due to trauma after the age of 74 years do not change significantly, although patients older than 74 years have twice the odds of death compared to those age 65-74 years..$^{19}$ Therefore, the true relationship between age and mortality may begin earlier than expected and progress nonlinearly. The literature reflects this ambiguity with a lack of consensus regarding the definition of old age, outcome variables, and statistical methods. Given these considerations, we sought to best characterize the incidence, etiology, injury severity, and outcomes in our population of geriatric patients with BCVI.

There was a lower incidence of BCVI in the geriatric group than in the adult group. If BCVI was present, falls and motor vehicle accidents were the most common etiology for geriatric and adult patients, respectively. Prior work by Page and Josiah and by Flashburg et al. corroborate the prevalence of falls and motor vehicle accidents in both age groups. ${ }^{7,8}$ National data from 2017 also support these findings. Falls are a leading cause of fatal injury and impaired quality of life, and 1 in 4 older adults report a fall each year. ${ }^{20}$ Unintentional injuries, including motor vehicle accidents, accounted for the most deaths in those younger than 45 years $(34.6 \%-40.6 \%)$, although such injuries accounted for only $2.7 \%$ of deaths in age groups 65 years and older. ${ }^{21}$ Furthermore, the absence of significant differences between individual screening criteria for either age group suggests that our criteria do not favor or neglect detection of BCVI in either age group. Thus, interventions aimed at reducing falls in high-risk patients with a history of falling, reduced strength, impaired gait, balance deficits, and polypharmacy ${ }^{22}$ will probably improve outcomes in the geriatric BCVI population and perhaps reduce its incidence in this at-risk population.

Despite a comparatively less severe mechanism of injury, grade IV BCVIs were significantly correlated with the geriatric group, whereas grade I injuries were associated with the adult group. This carries important prognostic and therapeutic implications. Biffl et al. showed that a higher injury grade significantly increases stroke risk $;^{6}$ further studies showed the increase to be as much as $21 \% .^{23}$ However, there was no significant difference in documented stroke between age groups in our study, nor in the study by Page and Josiah, that accounted for injury severity scores. ${ }^{8}$ The absence of a greater number of strokes despite a higher injury grade may initially suggest adequate intervention in the management of geriatric patients with BCVI who have higher injury grades. However, BCVI in elderly adults was associated not only with greater severity of injury, but also with worse prognosis. Although no difference was noted in the length of hospital or ICU stay, elderly adults tended to have worse outcomes, including death or discharge to skilled nursing facilities, whereas adults were discharged to home or self-care. Taken together, these outcomes suggest adequate prevention of stroke in elderly patients, although with greater mortality and morbidity following BCVI. The majority of BCVIs occurring in the setting of polytrauma points to the injury itself, rather than management, as being primarily responsible for the poor discharge disposition of geriatric patients.

The unfavorable outcomes of geriatric patients in the setting of seemingly benign etiologies may be explained by the physiological changes that accompany aging. Vascular damage, skeletal fragility, and comorbidities increase vulnerability of cerebrovascular vessels to lesser mechanisms of injury. Like the trend seen in trauma outcomes, vascular intimal changes appear to manifest in the 4th decade of life; by the age of 80 years, up to $80 \%$ of 
TABLE 4. BCVI outcomes by age group

\begin{tabular}{lrccc}
\hline \multicolumn{1}{c}{ Variable } & Overall & Adult & Geriatric & p Value \\
\hline Stroke on imaging, no. & & & & \\
\hline Yes & 7 & $6(85.7 \%)$ & $1(14.3 \%)$ & 0.7646 \\
\hline No & 114 & $93(81.6 \%)$ & $21(18.4 \%)$ & 0.9393 \\
\hline Not assessed & 2 & $1(50 \%)$ & $1(50 \%)$ & 0.2562 \\
\hline Unknown & 1 & $1(100 \%)$ & 0 & NA \\
\hline Disposition, no. & & & & \\
\hline Unspecified & 1 & $1(100 \%)$ & 0 & 0.6315 \\
\hline Died & 23 & $16(69.6 \%)$ & $7(30.4 \%)$ & 0.1489 \\
\hline Home or self-care & 27 & $27(100 \%)$ & 0 & 0.0127 \\
\hline Home w/ home healthcare services & 6 & $5(83.3 \%)$ & $1(16.7 \%)$ & 0.8984 \\
\hline Hospice/medical facility & 2 & 0 & $2(100 \%)$ & 0.0032 \\
\hline Long-term care & 20 & $18(90 \%)$ & $2(10 \%)$ & 0.3184 \\
\hline Rehabilitation facility & 26 & $22(84.6 \%)$ & $4(15.4 \%)$ & 0.6647 \\
\hline Skilled nursing home & 18 & $11(61.1 \%)$ & $7(38.9 \%)$ & 0.0280 \\
\hline
\end{tabular}

Percentages are reported with a denominator of the total number of patients in each variable (represented in rows). Boldface type indicates statistical significance.

vessels throughout the body may have a thickened intimal layer. ${ }^{24}$ The cessation of elastin production further stiffens arteries. ${ }^{24}$ Reduction in compliance and intimal damage result in decreased resilience and predispose arteries to endothelial disruption. ${ }^{24}$ Blunt trauma to the cervical region, through less severe mechanisms, may damage the intimal layer and consequently trigger thromboembolism, vessel stenosis, occlusion, or dissection.

Osteoporosis is a well-documented cause of morbidity and mortality in the geriatric population. Worldwide, it affects 1 in 3 women and 1 in 5 men older than 50 years. ${ }^{25}$ Additionally, a $10 \%$ loss of bone mass doubles the risk of vertebral fractures. ${ }^{25}$ Displaced or compressed bone fragments can easily damage the carotid and vertebral arteries that travel within the foramina or in close proximity. Although radiographically confirmed fractures of the upper cervical vertebral body, subluxation, and involvement of the transverse foramina have been shown to predict approximately $13 \%$ of $\mathrm{BCVIs},{ }^{26}$ spine fractures have a more subtle clinical presentation in the elderly. Schrag et al. found that tenderness in the neck region was the only accurate clinical predictor of the presence of cervical spine injury in geriatric patients with low-energy blunt trauma. ${ }^{27}$ Therefore, the predisposition toward fracture and subtle presentation can contribute to undesirable outcomes in the older age group.

The higher prevalence of comorbidities in the elderly may also help to explain the increased severity of outcomes after BCVI. A systematic review and meta-analysis for trauma patients quantified the direct association between the number of comorbidities and risk of death. ${ }^{28}$ Risk of death increased by 1.73 times and 2.72 times in the presence of 2 and 3 medical conditions, respectively. ${ }^{28}$ Centers for Disease Control and Prevention data in 2008 showed that $85.6 \%$ of individuals older than 65 years had at least 1 chronic condition and $56 \%$ of individuals had more than 2 chronic conditions. ${ }^{29}$ Although coexisting medical conditions can independently increase the likelihood of death, the aforementioned vascular and skeletal changes contribute to substantially higher mortality rates; even with survival, patients and their caretakers understandably experience a reduction in quality of life.

\section{Study Limitations}

The strength of this study is limited by several factors. Each patient entered into our database was screened with a predetermined set of signs, symptoms, and mechanisms. Although the severity of injury could have been misinterpreted with initial screening, all suspected BCVIs at our institution were confirmed radiographically. Interpretations were conducted by a trained neuroradiologist and secondarily verified during chart review. Additionally, although our data did not include Injury Severity Scores, prior studies that did include Injury Severity Scores have corroborated our findings. Our data did not include preinjury comorbidities, and further study is needed to determine how diabetes mellitus, atherosclerosis, hyperlipidemia, and smoking contribute to the epidemiology behind geriatric BCVIs. Likewise, our data are limited to shortterm outcomes. Future studies are warranted to assess the efficacy of interventions and long-term neurological outcomes and quality of life for BCVI survivors and their caretakers.

\section{Conclusions}

This study expands on prior work in geriatric BCVI by including carotid as well as vertebral arteries. We found that when carotid arteries were included, geriatric patients suffered a higher grade of injury from less severe mechanisms and a worse prognosis compared with the younger group. Asymmetrical anatomy may partially explain the laterality and mortality associated with carotid artery injuries. Age-associated vascular changes, skeletal disease, and concurrent medical conditions contribute to the greater severity and poor prognosis seen with BCVI in 
elderly patients. Communities and institutions that implement interventions aimed at addressing the unique needs of this vulnerable population should expect improvements in mortality and morbidity for a substantial proportion of their future population.

\section{References}

1. Tso MK, Lee MM, Ball CG, et al. Clinical utility of a screening protocol for blunt cerebrovascular injury using computed tomography angiography. J Neurosurg. 2017;126(4):10331041.

2. Bromberg WJ, Collier BC, Diebel LN, et al. Blunt cerebrovascular injury practice management guidelines: The Eastern Association for the Surgery of Trauma. J Trauma. 2010;68(2): 471-477.

3. Foreman PM, Harrigan MR. Blunt traumatic extracranial cerebrovascular injury and ischemic stroke. Cerebrovasc Dis Extra. 2017;7(1):72-83.

4. Burlew CC, Biffl WL, Moore EE, et al. Blunt cerebrovascular injuries: redefining screening criteria in the era of noninvasive diagnosis. J Trauma Acute Care Surg. 2012;72(2): 330-337, 539.

5. Miller PR, Fabian TC, Croce MA, et al. Prospective screening for blunt cerebrovascular injuries: analysis of diagnostic modalities and outcomes. Ann Surg. 2002;236(3):386-395.

6. Biffl WL, Moore EE, Offner PJ, et al. Blunt carotid arterial injuries: implications of a new grading scale. J Trauma. 1999; 47(5):845-853.

7. Flashburg E, Ong AW, Muller A, et al. Fall downs should not fall out: blunt cerebrovascular injury in geriatric patients after low-energy trauma is common. $J$ Trauma Acute Care Surg. 2019;86(6):1010-1014.

8. Page PS, Josiah DT. Traumatic vertebral artery injuries in the geriatric population: a retrospective cohort study. J Neurosurg Spine. 2020;32(5):657-660.

9. United States Census Bureau. Older people projected to outnumber children for first time in U.S. history. Census.gov. Accessed August 17, 2020. https://www.census.gov/newsroom/press-releases/2018/cb18-41-population-projections. html

10. Grossman A, Kanter D, Kleindorfer D, et al. University of Cincinnati Medical Center Guidelines on the Management of Blunt Cerebrovascular Injury (BCVI). February 7, 2018. Accessed August 17, 2020. http://todd-roat.squarespace.com/s/ UC-BCVI-Guidelines-030718.pdf

11. Harris PA, Taylor R, Thielke R, et al. Research electronic data capture (REDCap) - a metadata-driven methodology and workflow process for providing translational research informatics support. J Biomed Inform. 2009;42(2):377-381.

12. Harris PA, Taylor R, Minor BL, et al. The REDCap consortium: building an international community of software platform partners. J Biomed Inform. 2019;95:103208.

13. Esnault $\mathrm{P}, \mathrm{Cardinale} \mathrm{M}$, Boret $\mathrm{H}$, et al. Blunt cerebrovascular injuries in severe traumatic brain injury: incidence, risk factors, and evolution. J Neurosurg. 2017;127(1):16-22.

14. Ho AL, Deb S, Kim LH, et al. Predictors of cervical vertebral and carotid artery dissection during blunt trauma: experience from a level 1 trauma center. World Neurosurg. 2020;137:e315-e320.

15. Desai M, Rolls A, Girish G, Baker DM. Surgical approaches to the internal carotid artery at the extracranial middle skull base. Clin Surg. 2017;2:1551.

16. George B, Bruneau M, Spetzler RF, eds. Pathology and Surgery Around the Vertebral Artery. 1st ed. Springer; 2009.

17. Golemati S, Gastounioti A, Tsiaparas NN, et al. Bilateral asymmetry in ultrasound-image-based mechanical and textural features in subjects with asymptomatic carotid artery disease. In: IEEE-EMBS International Conference on Biomedical and Health Informatics (BHI). IEEE; 2014:680-683.
18. Adams SD, Cotton BA, McGuire MF, et al. Unique pattern of complications in elderly trauma patients at a Level I trauma center. J Trauma Acute Care Surg. 2012;72(1):112-118.

19. Hashmi A, Ibrahim-Zada I, Rhee P, et al. Predictors of mortality in geriatric trauma patients: a systematic review and meta-analysis. J Trauma Acute Care Surg. 2014;76(3): 894-901.

20. National Council on Aging. Falls prevention facts. NCOA. org. Accessed August 17, 2020. https://www.ncoa.org/news/ resources-for-reporters/get-the-facts/falls-prevention-facts/

21. Heron M. Deaths: Leading causes for 2017. National Vital Statistics Reports. NVSS. 2019;68(6):1-77.

22. Tinetti ME, Kumar C. The patient who falls: "It's always a trade-off." JAMA. 2010;303(3):258-266.

23. Cothren CC, Biffl WL, Moore EE, et al. Treatment for blunt cerebrovascular injuries: equivalence of anticoagulation and antiplatelet agents. Arch Surg. 2009;144(7):685-690.

24. Alvis BD, Hughes CG. Physiology considerations in geriatric patients. Anesthesiol Clin. 2015;33(3):447-456.

25. International Osteoporosis Foundation. Facts and statistics. Accessed August 17, 2020. https://www.iofbonehealth.org/ facts-statistics\#category-14

26. Kopelman TR, Leeds S, Berardoni NE, et al. Incidence of blunt cerebrovascular injury in low-risk cervical spine fractures. Am J Surg. 2011;202(6):684-689.

27. Schrag SP, Toedter LJ, McQuay N Jr. Cervical spine fractures in geriatric blunt trauma patients with low-energy mechanism: are clinical predictors adequate? Am J Surg. 2008; 195(2):170-173.

28. Nunes BP, Flores TR, Mielke GI, et al. Multimorbidity and mortality in older adults: a systematic review and meta-analysis. Arch Gerontol Geriatr. 2016;67:130-138.

29. Centers for Disease Control and Prevention. Percent of U.S. adults 55 and over with chronic conditions. CDC.gov. Accessed August 17, 2020. https://www.cdc.gov/nchs/health_ policy/adult_chronic_conditions.htm

\section{Disclosures}

The authors report no conflict of interest concerning the materials or methods used in this study or the findings specified in this paper.

\section{Author Contributions}

Conception and design: Prestigiacomo, Le, Ngwenya. Acquisition of data: Le, Barhorst, Castiglione, Harlan, Keegan. Analysis and interpretation of data: Prestigiacomo, Le, Barhorst, Castiglione, Yang, Shah, Jandarov, Ngwenya. Drafting the article: Le, Shah. Critically revising the article: Prestigiacomo, Le, Barhorst, Castiglione, Yang, Shah, Harlan, Keegan. Reviewed submitted version of manuscript: Prestigiacomo, Le, Barhorst, Castiglione, Yang, Shah, Harlan, Keegan. Approved the final version of the manuscript on behalf of all authors: Prestigiacomo. Statistical analysis: Le, Jandarov. Administrative/technical/material support: Prestigiacomo, Le, Ngwenya. Study supervision: Prestigiacomo, Le, Ngwenya.

\section{Supplemental Information \\ Online-Only Content}

Supplemental material is available online. Appendices $A$ and B. https://thejns.org/doi/suppl/10.3171/2020. 7.FOCUS20499.

\section{Correspondence}

Charles J. Prestigiacomo: University of Cincinnati College of Medicine, Cincinnati, OH. presticj@uc.edu. 